\title{
ESTUDIO COMPARATIVO DEL CONTENIDO DE COMPUESTOS BIOACTIVOS Y CIANIDINA-3- GLUCÓSIDO DEL MAÍZ MORADO (Zea mays L.) DE TRES REGIONES DEL PERÚ
}

\author{
Ana María Ccaccya Ccaccya ${ }^{* a}$, Mercedes Soberón Lozano ${ }^{\mathrm{b}}$, Inés Arnao Salas ${ }^{\mathrm{b}}$
}

\begin{abstract}
RESUMEN
El objetivo de la investigación fue evaluar el contenido de los compuestos bioactivos en coronta de maíz morado (Zea mays L.) de tres diferentes regiones del Perú. Las muestras fueron obtenidas en Lima, Abancay y Cajamarca y se determinó el contenido total de polifenoles, flavonoides, antocianinas y cianidina-3-glucósido. Los extractos fueron obtenidos con etanol al $20 \%, \mathrm{pH}$ 2, en una relación coronta: solvente 1:100 (p/v) a una temperatura de $70^{\circ} \mathrm{C}$ por 30 minutos. Los resultados de antocianinas totales fueron de 24,4 a $42,6 \mathrm{mg} / \mathrm{g}$, los polifenoles totales estuvieron en el rango de 67,9 a $94,1 \mu \mathrm{g} / \mathrm{mg}$ expresado como equivalente de ácido gálico (EAG) y los flavonoides entre 3,1 a 4,4 $\mu \mathrm{g} / \mathrm{mg}$ expresado como equivalente de quercetina (EQ). El contenido de cianidina-3-glucósido varió de 9,5 a $18,5 \mathrm{mg} / \mathrm{g}$ y fue determinado por cromatografía líquida de alta resolución (HPLC). Existen diferencias significativas en el contenido de los compuestos bioactivos según la procedencia de la muestra, y la de Lima presenta los valores más altos de polifenoles $(94,1 \mu \mathrm{g}$ EAG/mg), flavonoides $(4,4 \mu \mathrm{g} \mathrm{EQ} / \mathrm{mg})$, antocianinas totales $(42,6 \mathrm{mg} / \mathrm{g})$ y cianidina-3-glucósido $(18,5$ $\mathrm{mg} / \mathrm{g})$.
\end{abstract}

Palabras clave: maíz morado, compuestos bioactivos, antocianinas, cianidina-3-glucósido.

\section{COMPARATIVE STUDY OF THE CONTENT OF BIOACTIVES COMPOUNDS AND CYANIDIN-3-GLUCOSIDE OF THE PURPLE CORN (Zea mays L.) OF THREE REGIONS OF PERU}

\begin{abstract}
The aim of the research was to evaluate the content of the bioactives compounds in purple corn cob (Zea mays L.) of three differents regions of Peru. The samples were obtained in Lima, Cajamarca and Abancay and the total polyphenols, flavonoids, anthocyanins and cyanidin-3-glucoside content were determined. The extracts were obtained with etanol to 20 $\%, \mathrm{pH} 2$, in a ratio of corn cob: solvent $1: 100(\mathrm{p} / \mathrm{v})$ at a temperature of $70{ }^{\circ} \mathrm{C}$ for 30 minutes.

\footnotetext{
*a Facultad de Química e Ingeniería Química, UNMSM, Ciudad Universitaria, Av. Venezuela. Lima-Perú. maria_ jlas@yahoo.com

b Centro de Investigación de Bioquímica y Nutrición, Facultad de Medicina, UNMSM, Jr. Puno. Lima-Perú.
} 
The results of total anthocyanins were between 24,4 to $42,6 \mathrm{mg} / \mathrm{g}$, the total polyphenols were in the range 67,9 to $94,1 \mu \mathrm{g} / \mathrm{mg}$ expressed as gallic acid equivalent (GAE) and the flavonoids between 3,1 to $4,4 \mu \mathrm{g} / \mathrm{mg}$ expressed as quercetin equivalent (QE). The cyanidin3 -glucoside content varied in the range from 9,5 to $18,5 \mathrm{mg} / \mathrm{g}$ and was determined by high performance liquid chromatography (HPLC). There are significant differences in the content of the bioactives compounds according to the origin of the sample, and that of Lima (Cañete) present the highest value of polyphenols $(94,1 \mu \mathrm{g} \mathrm{GAE} / \mathrm{mg})$, flavonoids $(4,4 \mu \mathrm{g} \mathrm{QE} / \mathrm{mg})$ total anthocyanins $(42,6 \mathrm{mg} / \mathrm{g})$ y cyanidin-3-glucoside $(18,5 \mathrm{mg} / \mathrm{g})$.

Key words: purple corn, bioactives compounds, anthocyanins, cyanidin-3-glucoside.

\section{INTRODUCCIÓN}

El Perú es un país de gran biodiversidad y uno de sus recursos vegetales es el maíz morado (Zea mays L.) que presenta más de 35 variedades, pero todas ellas originadas a partir de la raza Kculli, que aún se sigue cultivando. Sus principales zonas de producción están en los departamentos de Cajamarca, Ayacucho, Ancash, Lima y Arequipa, siendo su ámbito de adaptación la costa y valles interandinos de la sierra hasta $3200 \mathrm{msnm}^{1}$.

El cultivo de maíz morado es valorado en el mercado nacional e internacional debido a su gran potencial nutricional y por su contenido de compuestos bioactivos (antocianinas y compuestos fenólicos). Investigaciones determinaron la presencia de ácidos fenólicos tales como el ácido vanílico, ácido cafeico y ácido ferúlico, además de otros flavonoides como hesperedina, quercetina y kaempferol ${ }^{2}$.

Muchas de las propiedades del maíz morado son atribuidas a su alto contenido de antocianinas, pigmentos que son responsables del color morado, rojo y azul de flores, hojas y frutos. Su consumo es de interés para la industria de colorantes alimenticios, debido a su capacidad para impartir colores atractivos y para la industria farmacéutica, por sus acciones benéficas para nuestra salud. Diversos estudios realizados demuestran que las antocianinas poseen actividad antioxidante, anticancerígena, antimicrobiana, antidiabética y antiflamatoria ${ }^{3}$.

La principal antocianina y responsable de las propiedades antioxidantes en el maíz morado es la cianidina-3-glucósido, la cual ha sido cuantificada por cromatografía líquida de alta resolución (HPLC) por Arriaga y Castro ${ }^{4}$, Pedreschi y Cisneros ${ }^{5}$, y Salinas et al. ${ }^{6}$ quienes han usado variedades de maíz morado procedentes de Cajamarca, Lima y México, respectivamente.

Teniendo en consideración que el contenido de los compuestos bioactivos depende de factores intrínsecos como la genética de la planta y factores extrínsecos como las condiciones ambientales del cultivo, nos propusimos comparar la concentración de polifenoles totales, flavonoides, antocianinas totales y cianidina-3-glucósido presentes en la coronta del maíz morado, procedente de tres diferentes regiones del Perú. 


\section{PARTE EXPERIMENTAL}

\section{Material vegetal}

Las corontas de maíz morado utilizadas en la presente investigación, se recolectaron de tres diferentes lugares del Perú entre los meses de julio 2017 a marzo 2018: Distrito de Pacarán, provincia Cañete de la región de Lima (700 msnm), distrito de Pichirhua, provincia Abancay de la región de Apurímac (2293 msnm) y en el distrito de Eduardo Villanueva, provincia San Marcos de la región de Cajamarca (2007 msnm). Todas las muestras fueron obtenidas de sus mismas zonas de cultivo.

\section{Preparación de la muestra}

La coronta de maíz morado fue previamente cortada en trozos pequeños, secado a $37{ }^{\circ} \mathrm{C}$ en una estufa INDUMELAB hasta peso constante; molido en una licuadora doméstica para finalmente pasarlo por una malla $\mathrm{N}^{\circ} 60$ y así obtener una muestra homogénea. Luego fue almacenado a temperatura ambiente en frascos oscuros.

Todos los reactivos empleados en la presente investigación fueron de grado analítico obtenidos de la casa Merck y Sigma Aldrich.

\section{Obtención de los extractos}

La extracción de los compuestos bioactivos de la coronta molida de maíz morado se realizó con etanol al $20 \%$, pH 2 ajustado con $\mathrm{HCl}$ concentrado, en una relación coronta: solvente 1:100 (p/v). Se colocó por 30 minutos a $70^{\circ} \mathrm{C}$ en baño maría BIOBASE y el extracto obtenido fue filtrado a través de papel Whatman $\mathrm{N}^{\circ} 40$, para luego proseguir con el ensayo.

\section{Métodos de análisis de los compuestos bioactivos}

\section{Determinación de antocianinas totales por método de pH diferencial}

El contenido de antocianinas totales fue determinado por el método de $\mathrm{pH}$ diferencial descrito por Giusti y Wrosltad ${ }^{7}$. Las diluciones fueron preparadas en buffer de cloruro de potasio $(\mathrm{pH}$ 1,0) y acetato de sodio ( $\mathrm{pH} 4,5$ ) empleando el potenciómetro METROHM. Las absorbancias fueron leídas en el espectrofotómetro GENESYS UV VIS a dos longitudes de onda $510 \mathrm{~nm}$ y $700 \mathrm{~nm}$.

Su contenido se expresó como cianidina-3-glucósido y se calculó de acuerdo a la siguiente expresión:

$$
\begin{gathered}
\mathrm{A}=\left(\mathrm{A}_{510 \mathrm{~nm}}-\mathrm{A}_{700 \mathrm{~nm}}\right)_{p H 1}-\left(\mathrm{A}_{510 \mathrm{~nm}}-\mathrm{A}_{700 \mathrm{~nm}}\right)_{p H 4,5} \\
\text { Antocianinas totales } \frac{\mathrm{mg}}{\mathrm{g}}=\frac{\mathrm{A}_{\times} \mathrm{MW}_{\times} \mathrm{FD}_{\times} \mathrm{V}}{\varepsilon_{\times} \mathrm{l}_{\times} \mathrm{W}}
\end{gathered}
$$


Donde:

MW (Peso molecular) $=449,2 \mathrm{~g} / \mathrm{mol}$ para cianidina-3-glucósido; FD = factor de dilución; 1 = longitud de paso de celda en $\mathrm{cm} ; \varepsilon=26900$ coeficiente de extinción molar para cianidina-3glucósido; $\mathrm{V}=$ volumen $\mathrm{mL}$ del solvente de extracción; $\mathrm{W}=$ peso en $\mathrm{g}$ de la muestra.

\section{Determinación de polifenoles totales}

El contenido de polifenoles se realizó conforme el procedimiento de la organización (ISO) 14502-1, usando un extracto etanólico de coronta de maíz morado al $0,5 \mathrm{mg} / \mathrm{mL}$. El tubo de reacción contenía $100 \mu \mathrm{L}$ del extracto, $500 \mu \mathrm{L}$ del reactivo Folin-Ciocalteu y $400 \mu \mathrm{L}$ de $\mathrm{Na}_{2} \mathrm{CO}_{3} 7,5 \%(\mathrm{p} / \mathrm{v})$ y las absorbancias fueron leídas a $765 \mathrm{~nm}$. Se realizó una curva de calibración empleando al ácido gálico como referencia en el rango de concentraciones de $0,5 \mathrm{a} 10 \mu \mathrm{g} / \mathrm{mL}\left(\mathrm{y}=0,09973 \mathrm{x}+0,01553, \mathrm{R}^{2}=0,999\right)$ y los resultados fueron expresados como equivalentes de ácido gálico (EAG) en $\mu \mathrm{g} / \mathrm{mg}$.

\section{Determinación de flavonoides}

La cuantificación de flavonoides se realizó según el procedimiento de Stalikas ${ }^{8}$, usando un extracto etanólico de coronta molida a $7 \mathrm{mg} / \mathrm{mL}$. El tubo de reacción se preparó mezclando: $100 \mu \mathrm{L}$ del extracto, $800 \mu \mathrm{L}$ de etanol y $100 \mu \mathrm{L} \mathrm{AlCl} 3$ al $20 \%(\mathrm{p} / \mathrm{v})$ y después de 30 minutos se leyó a $425 \mathrm{~nm}$. La medición se realizó empleando un blanco de muestra, en el cual se sustituyó el tricloruro de aluminio por etanol. Se preparó una curva de calibración empleando como referencia la quercetina en el rango de concentraciones de 0,5 a $14 \mu \mathrm{g} /$ $\mathrm{mL}\left(\mathrm{y}=0,07399 \mathrm{x}+0,00294, \mathrm{R}^{2}=0,999\right)$. Los resultados se expresaron como equivalentes de quercetina (EQ) en $\mu \mathrm{g} / \mathrm{mg}$.

\section{Determinación de cianidina-3-glucósido mediante HPLC}

Las condiciones de análisis fueron previamente descritas por Arriaga y Castro 4 . Se utilizó un equipo HPLC Hitachi con detector de arreglo de diodos. La separación se realizó en una columna Purospher STAR RP18 de $250 \mathrm{~mm}$ de longitud, diámetro 4,6mm y tamaño de partícula de $5 \mu \mathrm{m}$. La fase móvil estuvo compuesta de una solución A (ácido fórmico/agua/ metanol en proporción de 1:4:5) y una solución $\mathrm{B}$ (ácido fórmico/agua en una proporción de 1:9) y el gradiente de elución utilizado fue: $0 \min 10 \% \mathrm{~A}, 10 \min 73 \% \mathrm{~A}, 12 \min 73 \% \mathrm{~A}$, $20 \min 10 \% \mathrm{~A}$. Las lecturas fueron realizadas a $520 \mathrm{~nm}$. La identificación de la cianidina3-glucósido se realizó comparando el tiempo de retención de la muestra con el estándar y su cuantificación fue calculada en base a la relación de sus áreas. Las concentraciones de cianidina-3-glucósido se expresaron en $\mathrm{mg} / \mathrm{g}$ de coronta.

\section{Análisis Estadístico}

Todos los resultados se expresaron como promedio y desviación estándar de tres determinaciones independientes y fueron evaluados usando el paquete estadístico MINITAB 17. Para el análisis estadístico se aplicó ANOVA y el método de comparaciones Tukey. 


\section{RESULTADOS Y DISCUSIÓN}

\section{Determinación de antocianinas por el método de pH diferencial}

Las antocianinas son compuestos bioactivos presentes en el maíz y son responsables de los colores de este recurso nativo, por ello se espera que las variedades de pigmentación más intensa del maíz morado presenten una cantidad elevada de compuestos antociánicos. Uno de los factores que influye en su cuantificación es la eficacia de la extracción, lo cual depende de la naturaleza química de las antocianidinas, si están esterificadas tenderán a disolverse mejor en medio metanólico y si están glicosilada en medio acuoso 9 .

Los niveles de antocianinas de la coronta de maíz morado de las muestras ensayadas se presentan en la tabla 1 y varían entre 24,4 a $42,6 \mathrm{mg} / \mathrm{g}$, siendo mayor en la muestra obtenida del departamento de Lima.

En nuestro país existe información sobre la determinación de antocianinas en muestras procedentes de Cajamarca y de Arequipa. Resultados reportados por Rafael ${ }^{10}$ y Gorriti et $a l^{11}$ bajo condiciones de extracción similares a las desarrolladas en la presente investigación muestran valores de antocianinas de $8,7 \mathrm{mg} / \mathrm{g}$ y $25,9 \mathrm{mg} / \mathrm{g}$, respectivamente. Para el primer caso, la obtención de la coronta de maíz morado fue en el mercado de Cajamarca y no se conocen las condiciones de cosecha, recolección y almacenamiento que pudieran haber influido en la estabilidad de las antocianinas ya que Rafael ${ }^{10}$, menciona que la luz solar y sobre todo la temperatura pueden dar lugar a la hidrólisis del enlace glucosídico (posición 3) seguido de la ruptura del anillo y como efecto formación de chalconas incoloras. Esto podría explicar nuestros valores más altos de antocianinas ya que las muestras fueron obtenidas 3 días después de su cosecha e inmediatamente tratadas y almacenadas adecuadamente.

Por otro lado, Quispe et $a l^{12}$, con muestras obtenidas de Arequipa, reportaron valores de contenido de antocianinas entre 13,4 a $20,6 \mathrm{mg} / \mathrm{g}$, que fueron extraídas con etanol al 20\% pH 2 durante un tiempo de 60 minutos a la temperatura de $90{ }^{\circ} \mathrm{C}$, estos valores están por debajo de lo encontrado en nuestra investigación. Debemos mencionar que en nuestro laboratorio ensayamos la influencia del tiempo y temperatura, donde observamos que a temperaturas mayores a $70{ }^{\circ} \mathrm{C}$ y 30 minutos, el contenido de antocianinas disminuye. Estos resultados concuerdan con los estudios realizados por Gu et $a l^{13}$ donde demostraron que las antocianinas a temperaturas por encima de $\operatorname{los} 70^{\circ} \mathrm{C}$ se degradan siguiendo una cinética de primer orden y que dicha degradación térmica ocasiona la hidrólisis del enlace glucosídico, ruptura del anillo pirilio y formación de chalconas incoloras.

Ramos et al ${ }^{2}$ determinaron antocianinas en granos de maíz morado procedente de Cajamarca, para su extracción emplearon soluciones metanólicas ácidas a diferentes concentraciones obteniendo valores de 0,9 a 2,9 mg/g, que son significativamente menores a nuestro contenido de antocianinas de dicha región $(34,1 \mathrm{mg} / \mathrm{g})$. El mayor valor obtenido en nuestro estudio se justificaría por que en nuestra determinación usamos la coronta del maíz morado, donde es conocido que se concentra el mayor contenido de antocianinas, comparado con los granos de dicho fruto, hecho que fue confirmado por Arriaga y Castro ${ }^{4}$. 


\section{Determinación de polifenoles}

En la tabla 1, se presentan los resultados de la cuantificación de polifenoles para las tres diferentes regiones donde la muestra de Lima presenta el mayor contenido, con un valor de 94,1 $\mu \mathrm{g} \mathrm{EAG} / \mathrm{mg}$ de coronta.

Se ha determinado el contenido de polifenoles totales para muestras de corontas de maíz morado de la región Arequipa. Gorriti et a $l^{11}$ y Quispe et $a l^{12}$, encontraron valores de 77,0 $\mu \mathrm{g}$ EAG/mg y 40,6 $\mu \mathrm{g}$ EAG/mg respectivamente. Para el primer caso, la fecha de recolección fue entre los meses de febrero y abril, muy próxima a la de nuestra muestra de Cajamarca (entre febrero y marzo), lo que explicaría los valores cercanos. Para el segundo caso la recolección se hizo en noviembre y su resultado difiere totalmente de nuestras tres muestras investigadas. En ambos casos los factores que podrían haber influido en el contenido de polifenoles serían la altitud, condiciones climáticas, edafológicas y agronómicas.

Ramos et $a l^{2}$ cuantificaron los polifenoles en granos de maíz morado de Cajamarca y encontraron valores de 4,6 a 8,7 $\mu \mathrm{g}$ EAG/mg y Mex et al ${ }^{9}$ realizaron estudios de cinco variedades de maíces cultivados en México obteniendo el valor más alto en granos de maíz morado con 14,5 $\mu \mathrm{g}$ EAG/mg. En ambos estudios, los datos de polifenoles reportados en granos fueron inferiores a los de la coronta de maíz morado de nuestra investigación, lo cual también fue ya mencionado para el caso de las antocianinas, componente de este grupo químico.

\section{Determinación de flavonoides}

Existen pocos estudios sobre dicho análisis, uno de ellos es el de Ramos et al quienes trabajaron en granos de maíz morado de Cajamarca expresando sus resultados en equivalente de rutina (ER), en el rango de 1,2 a 2,6 $\mu \mathrm{g}$ ER/mg. En la presente investigación se usó la quercetina como referencia y obtuvimos de 3,1 a 4,4 $\mu \mathrm{g} \mathrm{EQ} / \mathrm{mg}$ de coronta, no siendo posible un análisis comparativo en equivalencia.

Tabla 1. Contenido de los compuestos bioactivos de la coronta del maíz morado de tres regiones del Perú.

\begin{tabular}{|c|c|c|c|c|}
\hline \multirow{3}{*}{$\begin{array}{l}\text { Provincia/ } \\
\text { Departamento }\end{array}$} & \multicolumn{4}{|c|}{ Compuestos Bioactivos } \\
\hline & Antocianinas & Polifenoles & Flavonoides & Cianidina-3- \\
\hline & Totales (mg/g) & $(\mu \mathrm{g}$ EAG/mg) & $(\mu \mathrm{g} \mathrm{EQ} / \mathrm{mg})$ & $\begin{array}{l}\text { glucósido } \\
(\mathrm{mg} / \mathrm{g})\end{array}$ \\
\hline Cañete (Lima) & $42,6 \pm 0,2^{\mathrm{a}}$ & $94,1 \pm 2,0^{\mathrm{a}}$ & $4,4 \pm 0,2^{\mathrm{a}}$ & $18,5 \pm 0,2^{\mathrm{a}}$ \\
\hline $\begin{array}{l}\text { San Marcos } \\
\text { (Cajamarca) }\end{array}$ & $34,1 \pm 0,2^{b}$ & $75,4 \pm 1,7^{b}$ & $3,6 \pm 0,1^{\mathrm{b}}$ & $10,3 \pm 0,1^{b}$ \\
\hline $\begin{array}{l}\text { Abancay } \\
\text { (Apurímac) }\end{array}$ & $24,4 \pm 0,3^{\mathrm{c}}$ & $67,9 \pm 2,2^{\mathrm{c}}$ & $3,1 \pm 0,1^{\mathrm{c}}$ & $9,5 \pm 0,1^{\mathrm{c}}$ \\
\hline
\end{tabular}




\section{Determinación de cianidina-3-glucósido mediante HPLC}

En la figura 1, los perfiles cromatográficos de las tres muestras son parecidos, variando sólo en la proporción de cada antocianina. Los picos predominantes fueron 1 y 3 , el primero fue identificado comparando el tiempo de retención del estándar cianidina-3-glucósido (figura 1A) con las tres muestras (figura 1B, 1C y 1D), para finalmente determinar su concentración comparando sus áreas respectivas. El pico 3, según Salinas et $a l^{6}$, es la cianidina-3-(6"malonilglucósido).

Según Pedreschi y Cisneros 5 , la cianidina-3-glucósido representa el principal tipo de antocianina presente en el extracto comercial de maíz morado obtenido de Lima, seguido por la cianidina-3-glucósido acilada. Asimismo, Cuevas et al ${ }^{14}$ investigaron sobre la caracterización de las antocianinas en granos de diferentes variedades de maíz boliviano, donde identificaron a la cianidina-3-glucósido y cianidina-3-(6"-malonilglucósido) como las mayoritarias, lo que concuerda con los resultados obtenidos en la presente investigación.
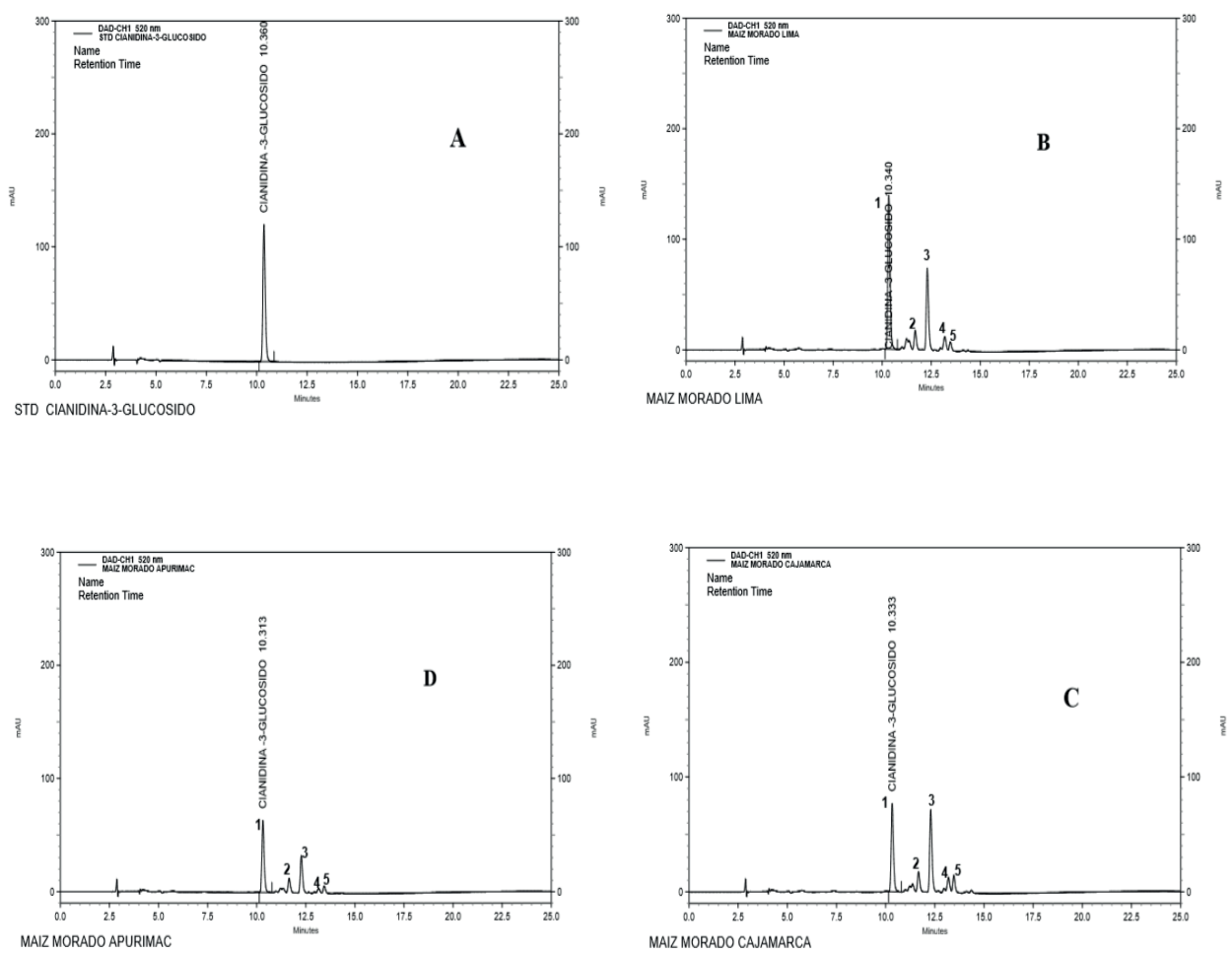

Figura 1. Cromatogramas A) Estándar de cianidina-3-glucósido B) Perfil obtenido del extracto de Lima C) Perfil obtenido del extracto de Cajamarca D) Perfil obtenido del extracto de Apurímac. 
En la tabla 1 se puede observar las concentraciones de cianidina-3-glucósido de diferentes regiones del Perú, donde se evidencia que la muestra de Lima presenta el mayor valor con un promedio de $18,5 \mathrm{mg} / \mathrm{g}$. Arriaga y Castro $^{4}$ estudiaron el contenido de cianidina-3-glucósido en coronta de seis muestras proporcionadas por el Instituto Nacional de Innovación Agraria y que fueron cultivadas en Cajamarca, donde concluyeron que la variedad identificada con el nombre de Sintético Amplio era la de mayor contenido $(30,8 \mathrm{mg} / \mathrm{g})$. Una probable explicación a este valor más alto, es que la muestra fue obtenida de un banco de germoplasma y fue genéticamente mejorada siendo por lo tanto las condiciones de cultivo diferentes a las especies de maíz morado de la presente investigación.

Por otro lado, Pedreschi y Cisneros ${ }^{5}$ determinaron el contenido de cianidina-3-glucósido en maíz morado de una muestra comercial proporcionado por Fitofarma (Lima), encontrando $15,4 \mathrm{mg} / \mathrm{g}$, siendo inferior al valor de nuestra muestra de Lima. Es necesario indicar que la muestra comercial correspondió a un extracto seco atomizado de maíz morado, además para su extracción se empleó la técnica de maceración en etanol al $60 \%$ por 48 horas a temperatura ambiente y tecnologías de secado por aspersión, mientras nosotros trabajamos con corontas de maíz morado bajo condiciones de laboratorio y condiciones de extracción diferentes.

Hernández et $a l^{15}$ realizaron la cuantificación de cianidina-3-glucósido en granos de maíz morado de diferentes variedades mexicanas obteniendo un máximo de $0,6 \mathrm{mg} / \mathrm{g}$, contenido que es muy inferior al rango de 9,5 a $18,5 \mathrm{mg} / \mathrm{g}$ que presentaron las 3 muestras de maíces peruanas, lo que confirma el menor valor de los compuestos bioactivos en granos en comparación con las corontas del maíz morado.

Finalmente, podemos mencionar que las variaciones en el contenido de los compuestos bioactivos del maíz morado en la presente investigación, podrían ser explicadas por diversos factores, entre estos tenemos, la fecha de recolección del recurso vegetal, las condiciones edafológicas, agronómicas, climáticas, diversidad genética, la naturaleza de la muestra, condiciones de extracción (efecto de tiempo, temperatura, concentración) entre otros. De estos factores, los 5 primeros tendrían una importante influencia en el maíz morado de Lima, por ser la muestra con mayor contenido de compuestos bioactivos.

\section{CONCLUSIÓN}

Los resultados de antocianinas totales, polifenoles totales, flavonoides y cianidina-3-glucósido de coronta de maíz morado procedente de Lima (Cañete) fueron los que presentaron los valores más altos, lo cual puede ser de interés para la industria alimentaria y farmacéutica. 


\section{AGRADECIMIENTOS}

Agradecimiento al Centro de Investigación de Bioquímica y Nutrición de la UNMSM por el apoyo brindado durante la realización de esta investigación.

\section{REFERENCIAS BIBLIOGRÁFICAS}

1. MINAGRI: Ministerio de Agricultura y Riego. Maíz morado. [Internet]; 2017 [citado 2 nov 2018]. Disponible en: http://repositorio.minagri.gob.pe/handle/MINAGRI/506

2. Ramos F, Muñoz AM, Alvarado C, Yáñez J. Purple corn (Zea mays L.) phenolic compounds profile and its assessment as an agent against oxidative stress in isolated mouse organs. J Med Food. 2012; 15(2): 206-215.

3. Aguilera M, Reza M, Chew R, Meza J. Propiedades funcionales de las antocianinas. Biotecnia. 2011; 13(2):16-22.

4. Arriaga B, Castro I. Determinación de la concentración de cianidina -3-glucósido por cromatografía líquida de alta resolución en Zea mays L. "maíz morado" procedente del Instituto Nacional de Innovación Agraria. [Tesis de Pregrado]. Trujillo: Universidad Nacional de Trujillo; 2011.

5. Pedreschi R, Cisneros L. Phenolics profiles of andean purple corn (Zea mays L.). Food Chem. 2007; 100: 956-963.

6. Salinas Y, Salas G, Rubio D, Ramos N. Characterization of anthocyanins extracts from maize kernels. J Chromatogr Sci. 2005; 43: 483-487.

7. Giusti MM, Wrosltad RE. Characterization and measurements of anthocyanins by UVVIS spectroscopy. CPFAC. 2001; F1.2.1-F.2.13.

8. Stalikas CD. Extraction, separation, and detection methods for phenolic acids and flavonoids. J Sep Sci. 2007; 30(18): 3268-3295.

9. Mex RMJ, Bolívar NJ, Garma PM, Tut JA, Romero KI. Actividad antioxidante de cinco variedades de maíz cultivadas en Campeche, México. Bol Latinoam Caribe Plant Med Aromat. 2013; 12(6):558-571.

10. Rafael E. Extracción y Cuantificación de Antocianinas de maíz morado (Zea mays L.) utilizando dos solventes a diferentes temperaturas y tiempos de extracción. [Tesis de pregrado]. Cajamarca: Universidad Nacional de Cajamarca; 2017.

11. Gorriti A, Arroyo J, Negrón L, Jurado B, Purizaca H, Santiago I, et al. Antocianinas, fenoles totales y actividad antioxidante de las corontas del maíz morado (Zea mays L.): Método de extracción. Bol Latinoam Caribe Plant Med Aromat. 2009; 8 (6): 509-518.

12. Quispe F, Arroyo K, Gorriti A. Características morfológicas y químicas de 3 cultivares de maíz morado (Zea mays L.) en Arequipa-Perú. Rev Soc Quim Perú. 2011; 73(3):205217.

13. Yang Z, Han Y, Gu Z, Fan G, Chen Z. Thermal degradation kinetics of aqueous anthocyanins and visual color of purple corn (Zea mays L.) cob. Innov Food Sci Emerg Technol. 2007; 9(3): 341-347.

14. Cuevas E, Antezana A, Winterhalter P. Análisis y caracterización de antocianinas en diferentes variedades de maíz morado (Zea Mays L.) boliviano. Memorias Red-Alfa Lagrotech. 2008; 79-95. 
15. Hernández J, Rosales A, Molina A, Miranda A, Willcox M, Hernández JM, et al. Cuantificación de antocianinas mediante espectroscopia de infrarrojo cercano y cromatografía líquida en maíces pigmentados. Fitotec. 2017; 40(2):219-225. 